\title{
The Rheological Properties of Asphalt Modified by Commercial Adhesive (Ethylene-Vinyl Acetate) and Air Blowing Process
}

\author{
Saad S. Ahmed ${ }^{1 *}$, Ammar A. Hamdoon ${ }^{2}$ \\ ${ }^{1}$ Nineveh Education Directorate, Ministry of Education, Mosul, Iraq \\ ${ }^{2}$ Department of Chemistry, College of Education for Pure Science, University of Mosul, Mosul, Iraq \\ E-mail: ${ }^{1 *}$ saadasalih3@gmail.com, ${ }^{2} \underline{\text { ammarhamdoon@ymail.com }}$
}

(Received February 09, 2020; Accepted May 03, 2020; Available online September 01, 2020)

DOI: 10.33899/edusj.2020.126673.1048, (c) 2020, College of Education for Pure Science, University of Mosul.

This is an open access article under the CC BY 4.0 license (http://creativecommons.org/licenses/by/4.0/).

\begin{abstract}
:
In this study, we modify the rheolgical prioperties of asphaltic materials by addative and air blowing. For this process, asphaltic materials have been oxidized by air blowing in the presence of anhydrous aluminum chloride as a catalyst for this process . Air blowing was conducted in different condtions of temperature and times using $0.5 \%$ (by weight) from catalyst, after determining the optimal conditions for air blowing, we changged the percentage of catalyst to get the optimal perecentage which can be using in modified process, then the asphalt was treated with the commercial adhesive (EVA) in two phaths:

The first path was included addtion of different percentages from commercial adhesive under the optimal condition from air blowing and perecentage of alaminum chloride.

The second path was included dissolved the commercial adhesive in the toluene and then the modified process was conducted at same conditions in the first path, ( toluene was added to the commercial adhesive by ratio (1:6) (weight of commercial adhesive: $\mathrm{ml}$ of toluene).

The results showe rheological properties that are compeletly different from the original asphaltic materials . The study was gave modified asphaltic materials that can be using in different fields, some samples can be using in paving as in samples $\left(\mathrm{AS}_{19}, \mathrm{AS}_{24}, \mathrm{AS}_{25}\right)$ and other as mastic or as flattening as in samples $\left(\mathrm{AS}_{15}, \mathrm{AS}_{16}, \mathrm{AS}_{17}, \mathrm{AS}_{21}, \mathrm{AS}_{22}\right)$. The usage of asphalitic materials in various fields depends on the measurments of different properties such as (ductility, penetration, softining point ,asphaltens percentage, penetration index ,aging test, marshall test).
\end{abstract}

keywords: Asphalt, Rheological Properties, air blowing

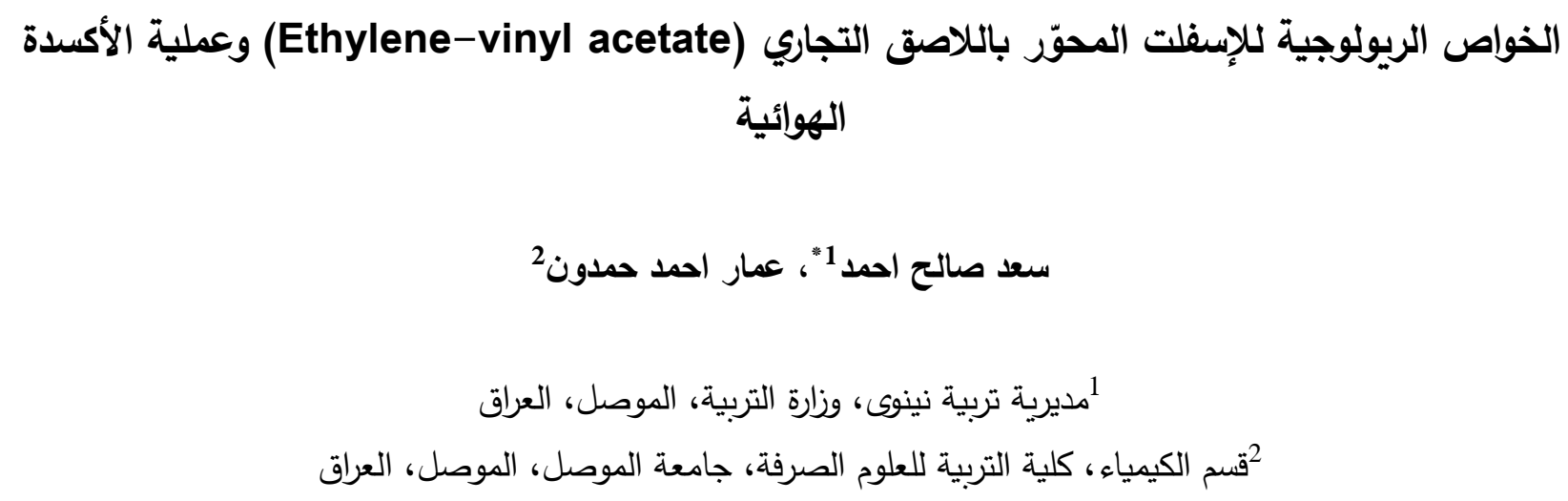


تضمنت هذه الدراسة تحوير الخواص الريولوجية للمواد الإسفلتية باستخدام المضافات والأكسدة الهوائية .تمت أكسدة المادة الإسفلتية

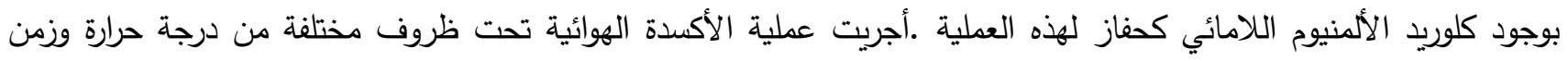
أكسدة بوجود (0.5 )\% وزنا من كلوريد الألمنيوم اللامائي .بعد تحديد الظروف المثلى لعملية الأكسدة الهوائية تم تغيير نسبة كلوريد

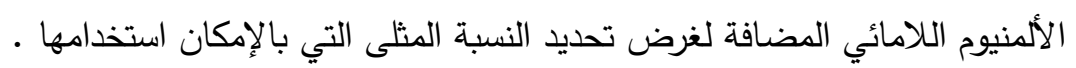

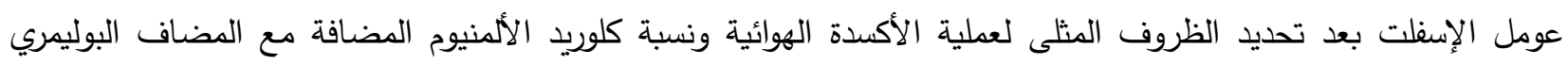

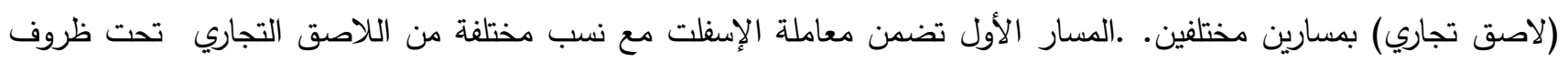
عملية الاكسدة المثلى. أما المسار الثاني فتم فيه استخدام اللاصق التجاري بهيئة مذابة في التولوين بنسبة (6:1)(وزن اللاصق : حجم المذيب) وتحت نفس الظروف المستخدمة في المسار ألأول من عملية أكسدة هوائية ونسبة حفاز .. أظهرت نتائج الدراسة إسفلت ذو خواص ريولوجية مختلفة تماما عن الإسفلت الأصل.،هاذ تم الحصول على نماذج اسفلتية

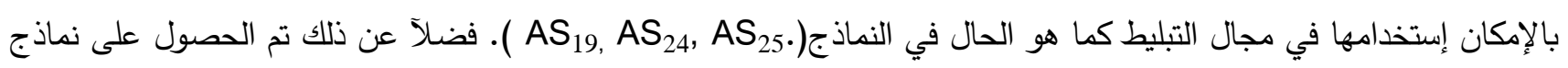
بالإمكان استخدامها كمواد مانعة للرطوبة وفي أعمال التسطيح كما هو الحال في النماذج( إن استخدام الإسفلت في مجالات مختلفة يتم تحديده عن طريق قياس العديد من المواصفات الريولوجية مثل ( الاستطالة , النفاذية , درجة الليونة , دليل الاختراق فحص المارشال والتقادم) فضلاً عن قياس النسبة المئوية للإسفلتين.

الكلمات المفتاحية: الإسفلت , الخواص الريولوجية, الأكسدة الهوائية

المقدمة

يعرف الاسفلت بأنه المكون الأتقل المستحصل عليه من عمليات التقطير المباشر للنفط الخام روهو اعقد المكونات ويزداد تعقيداً

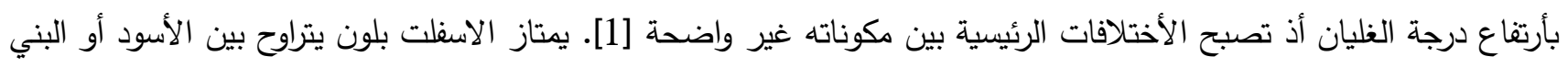

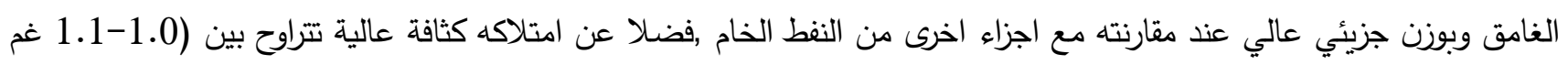

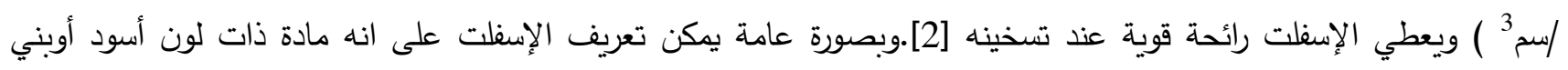

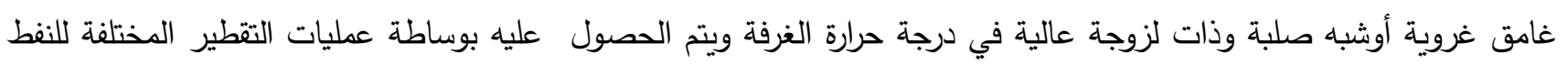
الخام [3,4].

وبسبب الخمول الكيميائي الذي يمتاز به الاسفلت والمترافق مع خواصه الفيزيائية جعل منه مؤهلا لاستعمالات واسعة في الصناعة والبناء منذ الأزل وان طبيعة الصفات الفيزيائية له هي التي تحدد طبيعة الاستعمال [2,5].

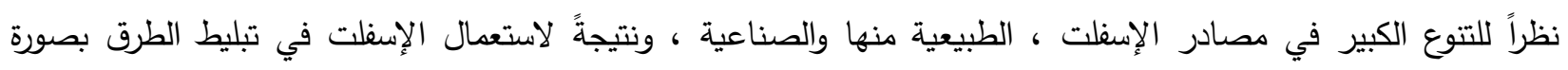
أساسية وغيرها من الإستعمالات الواسعة لكونه يتمتع بالتصاقية جيدة مع مختلف المواد الصلبة ، ولزوجته المناسبة ، وكذلك توفره بكلفة قليلة نسبياً لذلك أجريت عليه العديد من الدراسات .أظهرت تلك الدراسات أن خواصهه مثل اللزوجة والحساسية الحرارية يمكن أن تتحسن بوساطة استخدام المضافات المختلفة أو تحويره كيميائياً [6,7]. gالرجوع الى الدراسات السابقة نجد هناك عدد كثير من الدراسات التي تتتاول عملية تحوير الإسفلت منها ماقام به باستعمال الزيت المستهلك كمادة محورة وبنسب مختلفة تراوحت بين (1-12)\% وبأستخدام درجات حرارة منخفضة وأعطت الدراسة تحسناً في خواص الأسفلت تجاه عملية الأنتزاع و القشط ـ ودرس كل من Arslin Toque [9 الخواص الريولوجية للإسفلت 
بمعاملته مع نسب مختلفة من زيت التزييت بالتسخين وكان هناك تغير إيجابي واضح بالقياسات من درجة الليونة روالنفاذية وقابلية

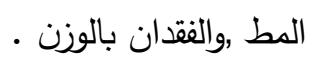

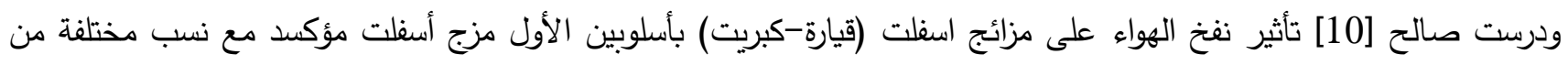

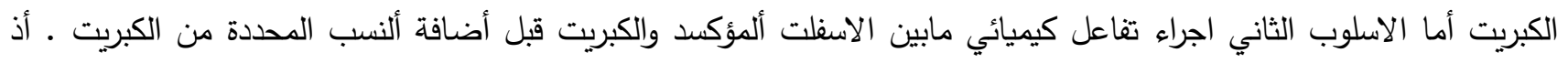

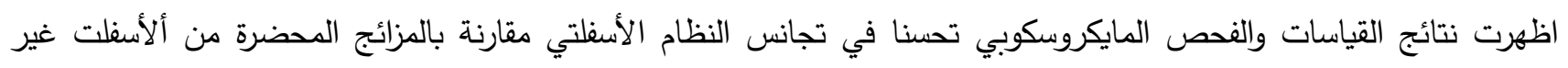
المحور. كما قام كل من Hussein Mohammed [11] بتحسين أداء خصائص الأسفلت بأستخدام تيرفثالات الأثلين (PET) اذ درست

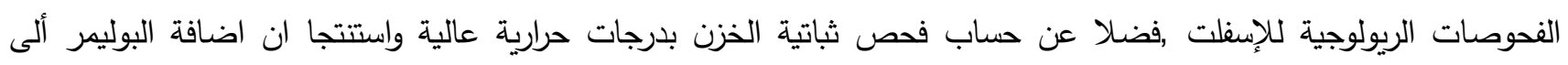

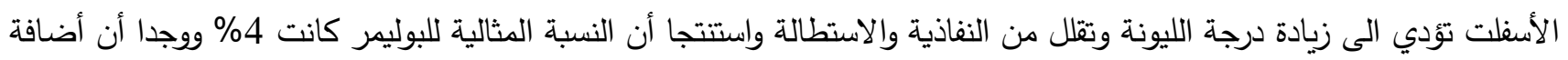
ألبوليمر تؤدي الى تقليل ألحساسية الحرارية. كما تمكن Gama [12] وجماعته من دراسة الخواص الريولوجية للإسفلت المحوَّر بالبوليمرات من نوع (إيثلين-ميثايلـاكريليت(EMA - GMA) كلاسيديل-ميثا أكريليت) (Ethylene- Methyl - Acrylate- Glycidyl- Methacrylate)

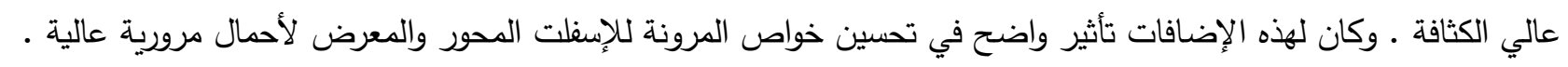

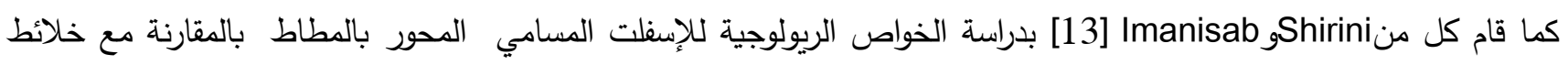
الإسفلت المسامي المحور بال(SBS) ووجدا ان اضافة 10\% من فتات المطاط ادى الى تحسين اداء الإسفلت المسامي في مقاومة التعفن مقارنة مع الاسفلت المحور بال SBS (ستايرين_بيوتادايين_ستايرين).

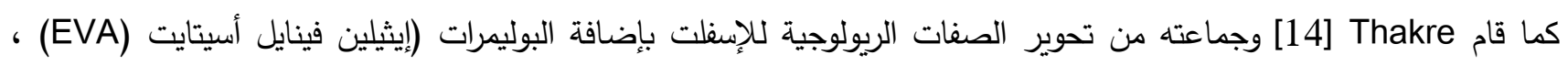

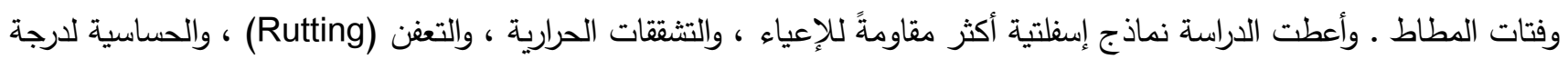
الحرارة • وبالإمكان استعمالها في مجال التبليط .

كما تمكن الباحثان حسين وحمدون [15] من تحوير الخواص الريولوجية للإسفلت باستعمال الزيوت المستهلكة والأكسدة الهوائية وقد تم الحصول من خلال هذه الدراسة على نماذج إسفلتية ذات مواصفات ريولوجية بالإمكان استعمالها في مجال التبليط وأخرى يمكن استعمالها كمواد مانعة للرطوبة اعتمادا على القياسات التي تم إجراؤها (الاستطالة والنفاذية و درجة الليونة و نسبة الإسفلتين فضلانلا دئل

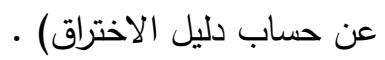

\author{
الجزي العملي \\ Materials Used \\ اولا- المواد المستعملة \\ 1- إسفلت الدورة \\ تم الحصول عليه من مصفى الدورة الذي يمتاز بالمواصفات الموضحة في الجدول(1):
}




$$
\text { جدول(1) الخواص الريولوجية لإسفلت الدورة }
$$

\begin{tabular}{|c|c|}
\hline القيمة المختبرية & المواصفات \\
\hline $150<$ & الاستطالة (25,cmمم) \\
\hline 50 & درجة الليونة(مº) \\
\hline 45.3 & النفاذية ,ملم (100غم,5ثا,25م²) \\
\hline-1.413 & دليل الاختراق(PI) \\
\hline 19.2 & نسبة الإسفلتين\% \\
\hline
\end{tabular}

2-كلوريد الألمنيوم اللامائي VWR Chemicals هكسان اعتيادي (n- Hexane) مجز من شركة

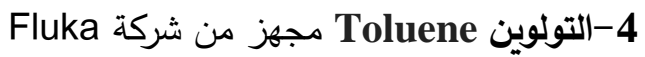

$$
\text { 5-لاصق تجاري(EVA):تم الحصول عليه من الاسواق المحلية }
$$

Instruments Used

1-جهاز قياس الاستطالة Ductility يعمل هذا الجهاز على قياس المسافة التي تستطيل بها المواد الإسفلتية عند تعرضها لتأثير سحٍ وبسرعةٍ ثابتة ، إلى ان ينقطع الأنموذج الإسفلتي. والجهاز ذو منشأ صيني من نوع (YUFENG)

2-جهاز قياس النفاذية Penetromete يعمل هذا الجهاز على فحص نفاذية المواد البتيومينية الصلبة وشبـه الصلبـة. فالنفاذيـة هـي مقياس لصلابـة الإسفلت والجهاز ذو منشأ صيني نوع (YUFENG) .

3- جهاز قياس درجة الليونة Ring and Ball Apparatus يعمل هـذا الجهـاز على قيـاس درجة الليونة للمواد الإسفلتية

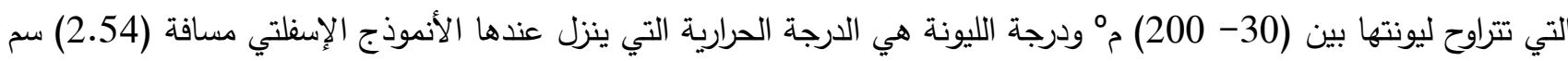

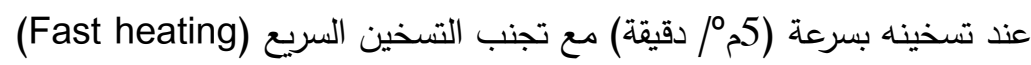

Polymer Treated Asphalt Apparatus جهاز معالجة الإسفلت بالمضافات

5-جهاز ضخ الهواء Air Blowing Apparatus وهومن نوع Dawson McDonald and Dawson 6- جهاز الرج الكهربائي Electrical Shaker وهو من نوع Hamber Ggo Shaker Germany

7 - جهاز اختبار المارشال Marshall Testing Apparatus هذا الفحص يعطي دلالة عن مدى ملائمة الإسفلت للتبليط: والجهاز ذو منشأ إنكليزي نوع (WYKEHAM FARRANCE) • 


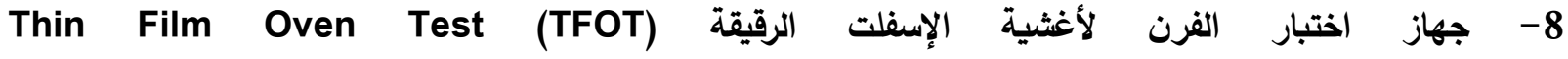

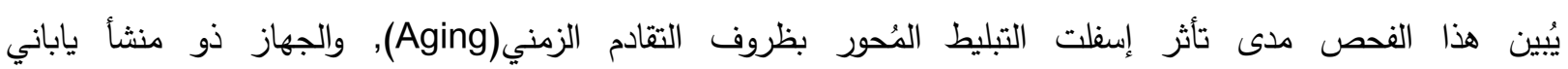
موديل 710812

\section{Experimental Methods}

ثالثا:الطرائق العملية

\section{Air Oixidation of Asphalt}

1-الأكسدة الهوائية للأسفلت

أُخذ وزن معين من الإسفلت ووضع في جهاز معالجة المادة الإسفلتية ، ومرر عليه الهواء من جهاز ضخ الهواء وبمعدل سرعة

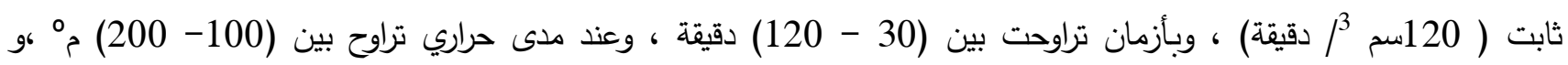
باستخدام (0.5 )\% وزنا من كلوريد الالمنيوم اللامائي كحفاز لهذه المعاملة. 2- تحديد النسبة المثلى للحفاز

بعد تحديد الزمن ودرجة الحرارة المثلى لعملية الاكسدة وأعتماداً على الخطوة (1) تم تغير نسبة الحفاز المضافة، اذ استخدمت

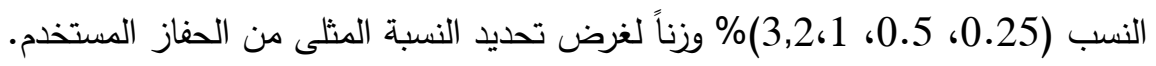
3- معاملة الإسفلت مع اللاصق التجاري(EVA) وبمسارين :

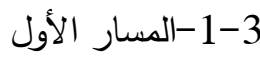
عومل الإسفلت مع نسب مختلفة من اللاصق التجاري تراوحت بين (1 - 5)\% وزنا عند درجة حرارة 150مْ وبوجود 0.5\% وزنا من كلوريد الامنيوم اللامائي لمدة 60 دقيقة وبعملية أكسدة هوائية والتي تمثل الظروف المثلى التي تم الحصول عليها من الخطوتين(1)(2). 3-2-3-المسار الثاني

عومل الإسفلت تحت نفس الظروف المذكورة في المسار الاول مع نسب مختلفة من اللاصق المذاب في التولوين بنسبة (6:1)

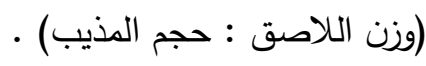

\section{Asphaltene separation}

4-فصل الإسفلتين

جميع النماذج المحضرة فضلا عن الأصل أجريت عليها عملية فصل الاسفلتين , إذ وضع في دورق زجاجي غرام واحد من نماذج الإسفلت المختلفة ، وأضيف إليها (40) مل من الهكسان الإعتيادي بنسبة (1 :40) (وزن: حجم) • وزُج المحلول باستخدام جهاز الرج الكهربائي لمدة ثلاث ساعات في درجة الصفر المئوي ـ ثم فصل الإسفلتين عن طريق ترشيح المزيج • إذ يمثل الإسفلتين

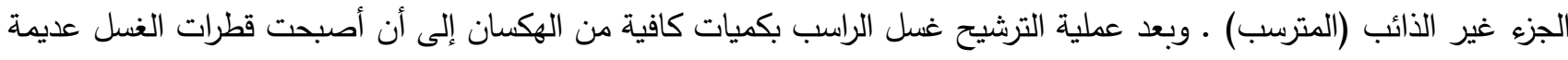

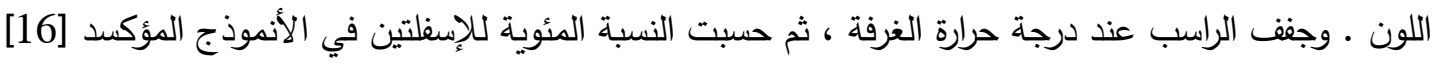
5-تحديد الخواص الريولوجية للأسفلت الأصل والمحور

تم تحديد المواصفات الريولوجية للمادة الاسفلتية الاصل والمعاملة وشمل ذلك قياس كل من الاستطالة [17] ودرجة الليونة [18] والنفاذية [19] وحساب دليل الاختراق لجميع النماذج [20] ,فضلا عن قياس المارشال [21] والتقادم [22] لبعض التصني النتائج.

\section{النتائج والمناقشة}

تعد عملية الحصول على اسفلت ذي خواص ريولوجية مطابقة لما هو موجود في المواصفات القياسية سواء كان استخدامه في مجال التبليط او التسطيح او العزل وما ألى ذلك من أهم الامور التي سعى اليها الباحثون من خلال استخدام طرق تحوير مختلفة 
. في دراستنا هذه تم استخدام عملية الاكسدة الهوائية مع إضافة لاصق تجاري متوفر بكثرة في الاسواق المحلية كمادة مُحورة اساسية. للإسفلت • يتكون هذا اللاصق بشكل عام من (EVA) (Ethylene-vinyl acetate)

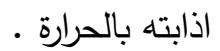
أجريت عملية الاكسدة الهوائية وكما هو موضح في الفقرة ثالثا من الجزء العملي لغرض تحديد الظروف المثلى لعملية التحوير

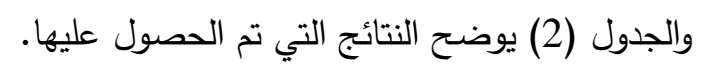

الجدول (2) : الخواص الريولوجية للإسفلت المؤكسَد هوائياً عند درجات حراةٍ وأزمانٍ مختلفة وبوجود 0.5\% وزناً من كلوريد الألمنيوم

\begin{tabular}{|c|c|c|c|c|c|c|c|}
\hline \multicolumn{8}{|c|}{ اللامائي } \\
\hline الإسفلتين\% & $\begin{array}{c}\text { الاخترلق } \\
\text { (PI ) }\end{array}$ & (100 غم,5ثا, 25مثم) & الليونة (مثم) & $\begin{array}{c}\text { الاستطالة } \\
\text { (25,cm) }\end{array}$ & الحرارة & (لزقيقة) & الأنموذج \\
\hline 19.2 & -1.413 & 45.3 & 50 & $150<$ & 0 & 0 & $\mathrm{AS}_{0}$ \\
\hline 23.3 & +0.387 & 41.6 & 59 & 85 & 100 & 30 & $\mathrm{AS}_{1}$ \\
\hline 25.6 & +0.705 & 40.3 & 62 & 110 & 100 & 60 & $\mathrm{AS}_{2}$ \\
\hline 28.3 & +0.986 & 38.7 & 63 & 96 & 100 & 90 & $\mathrm{AS}_{3}$ \\
\hline 30.4 & +1.599 & 37.2 & 68 & 65 & 100 & 120 & $\mathrm{AS}_{4}$ \\
\hline 21.7 & +0.320 & 40.4 & 59 & 93 & 150 & 30 & $\mathrm{AS}_{5}$ \\
\hline 23.2 & -0.025 & 41.5 & 57 & $150<$ & 150 & 60 & $\mathrm{AS}_{6}$ \\
\hline 25.5 & +0.848 & 39.5 & 62 & 90 & 150 & 90 & $\mathrm{AS}_{7}$ \\
\hline 27.3 & +0.896 & 37.2 & 63 & 74 & 150 & 120 & $\mathrm{AS}_{8}$ \\
\hline 27.4 & +0.833 & 40.1 & 62 & 83 & 200 & 30 & $\mathrm{AS}_{9}$ \\
\hline 32.6 & +0.681 & 36.7 & 62 & 79 & 200 & 60 & $\mathrm{AS}_{10}$ \\
\hline 29.1 & +1.028 & 36.4 & 64 & 65 & 200 & 90 & $\mathrm{AS}_{11}$ \\
\hline 36.2 & +1.303 & 35.2 & 66 & 52 & 200 & 120 & $\mathrm{AS}_{12}$ \\
\hline
\end{tabular}

AS 


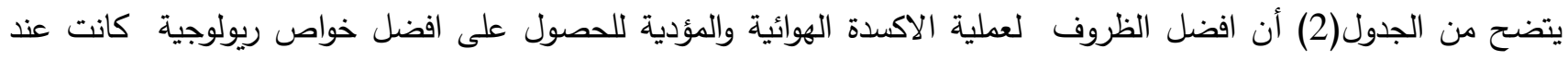

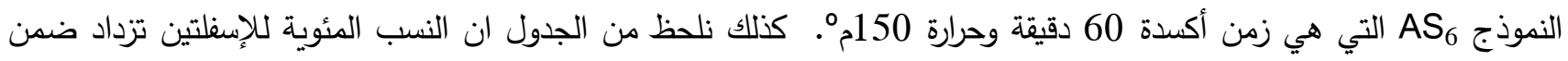
درجة الحرارة الواحدة بازدياد زمن الاكسدة الى حد معين ثم تبدأ بالأنخفاض عند الزمن الأعلى ,ان هذا الانخفاض في قيم الإسفلتين

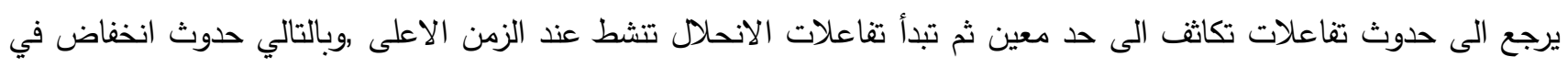
النسبة المئوية للأسفلت.

بعد تثبيت الظروف المثلى لعملية الاكسدة الهوائية , تم تحديد النسبة المثلى للتحفيز وكما هو موضح في الفقرة ثالثا من الجزء العملي والجدول (3) يوضح النتائج التي تم الحصول التعلئ عليها.

جدول (3) :الخواص الريولوجية للإسفلت المعامل مع نسب مختلفة من كلوريد الالمنيوم اللامائي عند 150مْ وزمن ساعة وبعملية اكسدة هوائية

\begin{tabular}{|c|c|c|c|c|c|c|}
\hline |الإسفلتين\% & $\begin{array}{l}\text { دليل الاخترلق } \\
\text { (PI ) }\end{array}$ & 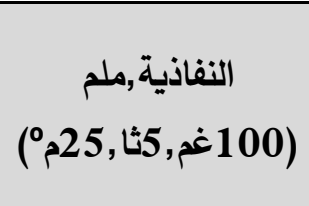 & الليونة (مُم) & $\begin{array}{c}\text { الاستطالة } \\
\text { (25,cm) }\end{array}$ & ألحفاز & الأنموذج \\
\hline 19.2 & -1.413 & 45.3 & 50 & $150<$ & 0 & $\mathrm{AS}_{0}$ \\
\hline 26.3 & +0.414 & 38.6 & 60 & 91 & 0 & AS* \\
\hline 24.2 & +0.699 & 40.2 & 61 & 110 & 0.25 & $\mathrm{AS}_{13}$ \\
\hline 23.2 & -0.025 & 41.5 & 57 & $150<$ & 0.5 & $\mathrm{AS}_{14}$ \\
\hline 26.7 & +0.663 & 36.4 & 62 & 58 & 1 & $\mathrm{AS}_{15}$ \\
\hline 29.4 & +1.225 & 34 & 66 & 32 & 2 & $\mathrm{AS}_{16}$ \\
\hline 29.6 & +0.836 & 33.4 & 64 & 31 & 3 & $\mathrm{AS}_{17}$ \\
\hline
\end{tabular}

النموذج الاصل دون أي معاملة

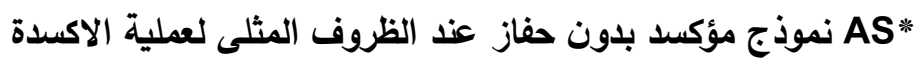

نلحظ من الجدول أعلاه أن نسبة الحفاز التي تم اختيارها في بداية التفاعل والتي هي (0.5\%) وزناً، تمثل أفضل نسبةٍ يمكن استخدامها لتحوير الخواص الريولوجية للإسفلت باستخدام عملية الأكسدة الهوائية. يتضح من الجدول (2) والجدول (3) ان أفضل الظروف لتحوير لتحوير ألإسفلت باستخدام عملية الاكسدة الهوائية عند زمن (60)

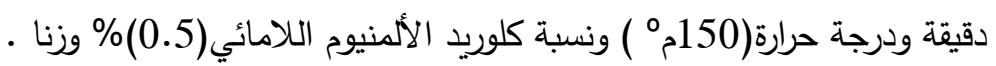
استخدمت هذه الظروف في معاملة الإسفلت مع نسب مختلفة من اللاصق التجاري (EVA) والجدول (4) والثكل (1) يوضحان النتائج التي تم الحصول عليها . 
جدول(4) : الخواص الريولوجية للإسفلت المعامل مع نسب مختلفة من اللاصق التجاري (EVA)عند 150مْ وزمن ساعة بوجود 0.5\% وزنا من كلوريد الالمنيوم اللامائي بعملية اكسدة هوائية.

\begin{tabular}{|c|c|c|c|c|c|c|}
\hline $\begin{array}{c}\text { الإسفلتين } \\
\text { \% }\end{array}$ & $\begin{array}{l}\text { الاخترلق دليل } \\
\text { (PI) }\end{array}$ & (100 أنفاذية, 5ثا, 25مُ & الليونة (مº & 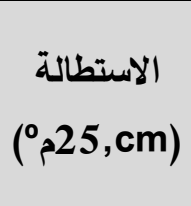 & $\begin{array}{c}\text { نسبة اللاصق } \\
\text { \%(EVA) }\end{array}$ & الأنموذج \\
\hline 19.2 & -1.413 & 45.3 & 50 & $150<$ & 0 & $\mathrm{AS}_{0}$ \\
\hline 21.2 & -0.361 & 43.2 & 54 & $150<$ & 1 & $\mathrm{AS}_{18}$ \\
\hline 24.6 & -0.172 & 42.7 & 56 & $150<$ & 2 & $\mathrm{AS}_{19}$ \\
\hline 25.1 & +0.183 & 38.7 & 59 & 130 & 3 & $\mathrm{AS}_{20}$ \\
\hline 29.3 & +0.583 & 37.8 & 61 & 74 & 4 & $\mathrm{AS}_{21}$ \\
\hline 30.4 & +1.089 & 37.4 & 64 & 62 & 5 & $\mathrm{AS}_{22}$ \\
\hline
\end{tabular}

AS

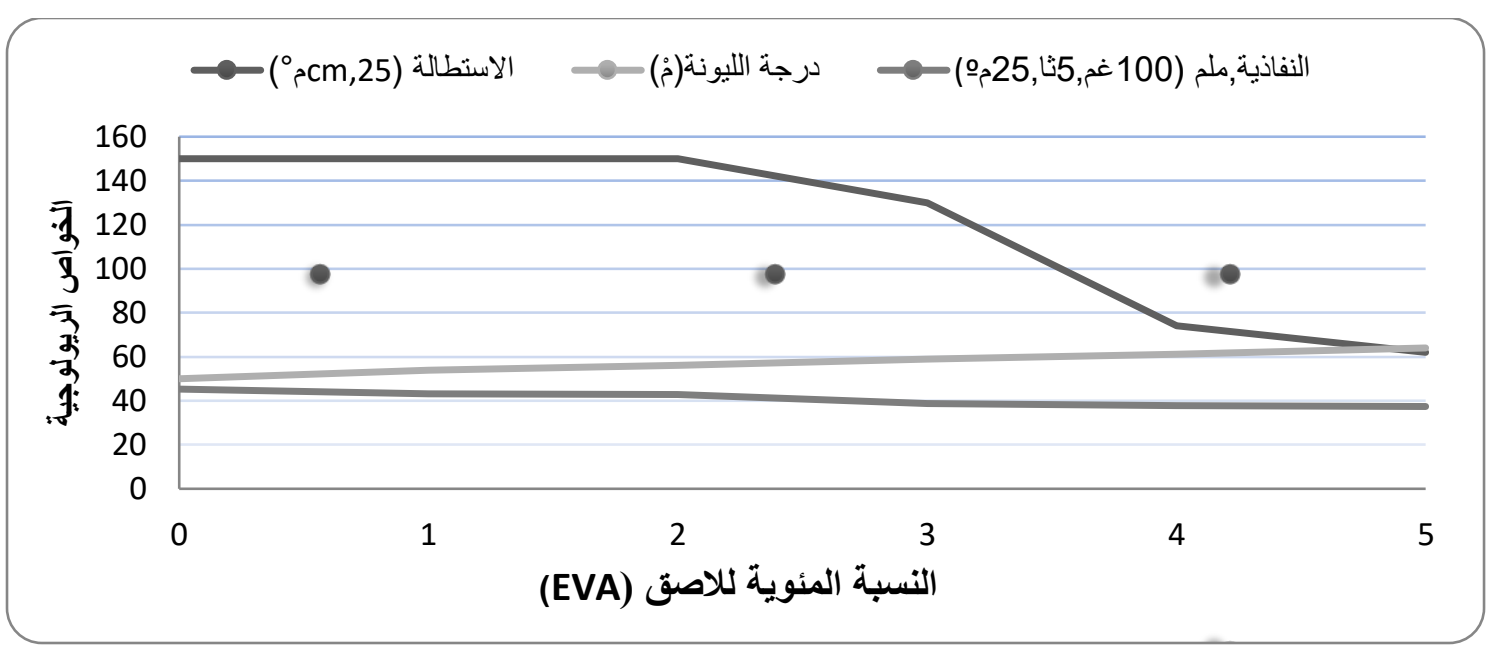

الثكل(1) تأثير إضافة اللاصق التجاري(EVA )على خواص الإسفلت الريولوجية 
يتضح من الجدول (4) أن عملية استخدام المادة اللاصقة جنبا الى جنب مع عملية الأكسدة الهوائية كان اختيارا جيدا في ضوء

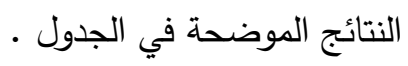
كذلك نلحظ من الجدول ان قيم الاستطالة للنماذج المحورة كانت ضمن حدود المواصفات القياسية لإسفلت التبليط الموضحة في

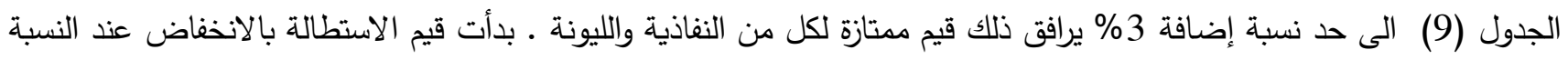

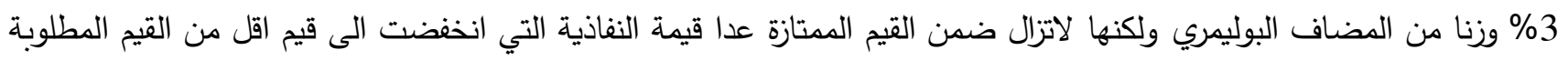
.اما النسب 5,4\% فأنها أدت الى انخفاض قيم الاستطالة بنسب اكبر مما هو عليه عند النسبة 3\% و وبمدى خارج المواصفات المطلوبة لإسفلت التبليط .

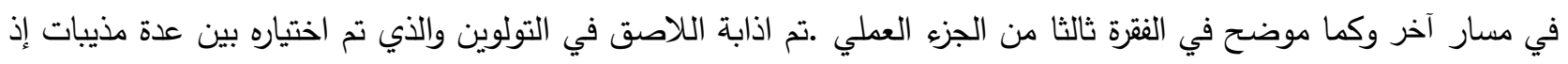
اتضح ان أفضلها في الإذابة ـ وأعيد استخدام اللاصق التجاري المذاب في التولوين في عملية تحوير الخواص الريولوجية للإِسفلت وتحت الظروف المثلى لعملية الاكسدة الهوائية والجدول (5) والثكل (2) يوضحان النتايج التي تم الحصول عليها.

جدول (5)

الخواص الريولوجية للاسفلت المعامل مع نسب مختلفة من اللاصق (EVA)(المذاب في التولوين بوجود 0.5\% وزنا من كلوريد الالمنيوم اللامائي عند 150 مْ وزمن ساعة وبعملية اكسدة هوائية.

\begin{tabular}{|c|c|c|c|c|c|c|}
\hline الإسفلتين\% & الاخترلق دليل & (100 النفاذية,ملم,5ثا, 25مم) & الليونة (مُم) & 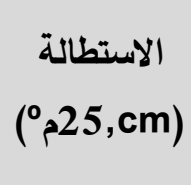 & $\begin{array}{l}\text { نسبة اللاصق } \\
\text { \%(EVA) }\end{array}$ & الأنموذج \\
\hline 19.2 & -1.413 & 45.3 & 50 & $150<$ & 0 & $\mathrm{AS}_{0}$ \\
\hline 23.8 & -1.012 & 43.7 & 52 & $150<$ & 1 & $\mathrm{AS}_{23}$ \\
\hline 26.7 & -0.592 & 42.9 & 54 & $150<$ & 2 & $\mathrm{AS}_{24}$ \\
\hline 27.8 & -0.450 & 41.5 & 55 & $150<$ & 3 & $\mathrm{AS}_{25}$ \\
\hline 28.3 & +0.303 & 40.1 & 59 & 145 & 4 & $\mathrm{AS}_{26}$ \\
\hline 29.2 & +0.819 & 39 & 62 & 80 & 5 & $\mathrm{AS}_{27}$ \\
\hline
\end{tabular}

AS 


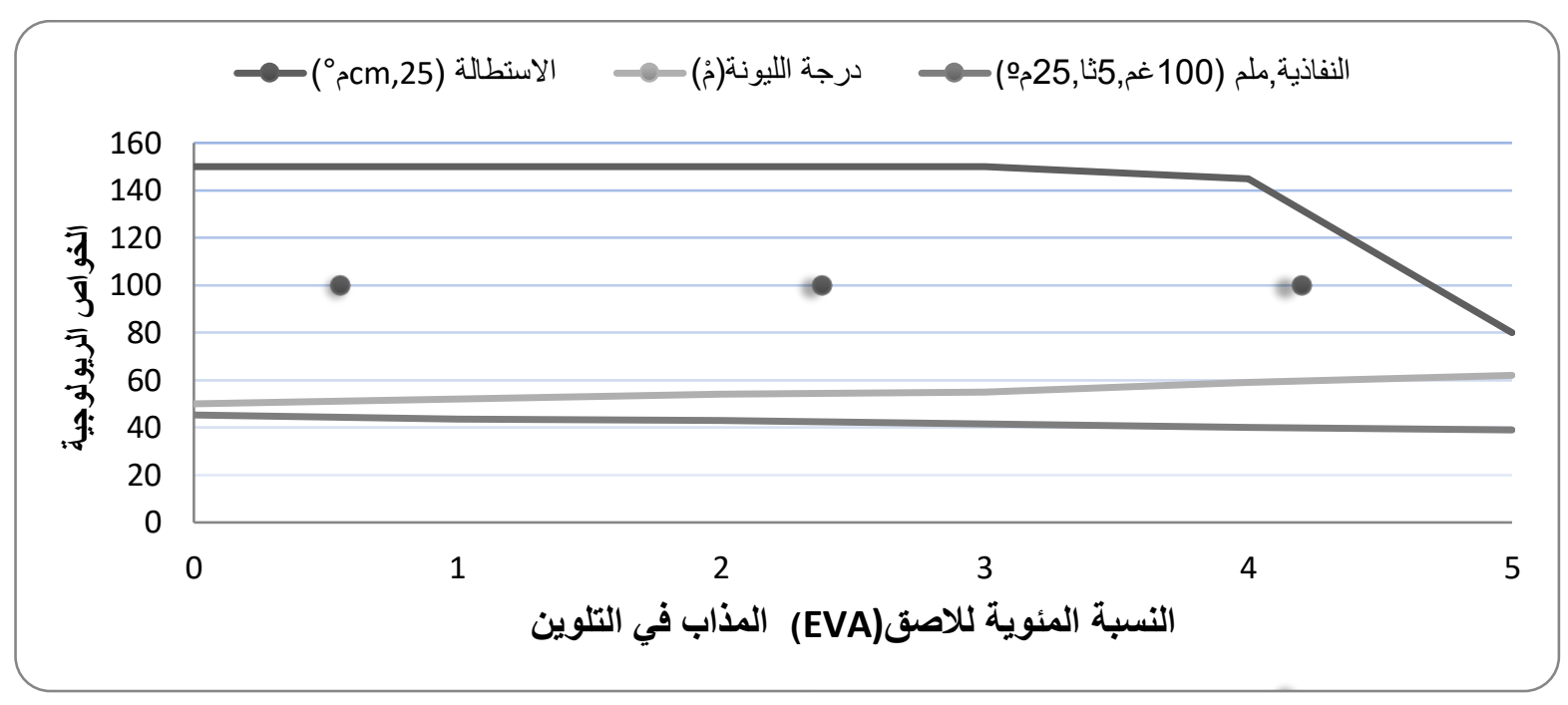

الثكل(2) تأثير اضافة اللاصق التجاري(EVA) المذاب في التولوين على خواص الاسفلت الريولوجية

نلاحظ من الجدول أعلاه ان عملية استخدام اللاصق الدذاب أدت الى زيادة نسبة المضاف المستخدم بالتحويرألى حد النسبة 4\% من المضاف (من حيث قيم الاستطالة والنفاذية والليونة والتي كانت ضمن المدى المسموح به للنماذج القياسية ) ,حيث كانت 3.

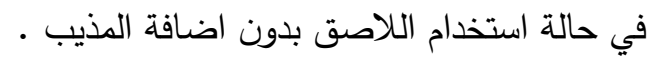
إن هذا التغير في الخواص الريولوجية عند استخدام اللاصق المذاب بألتاكيد عائد الى ان اللاصق ( المذاب ) يحقق امتزاجاً بصورة

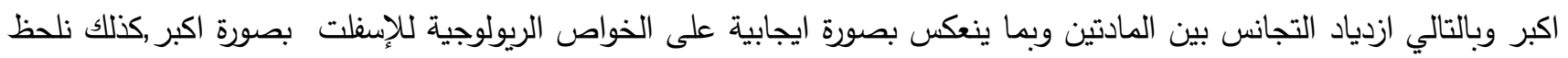

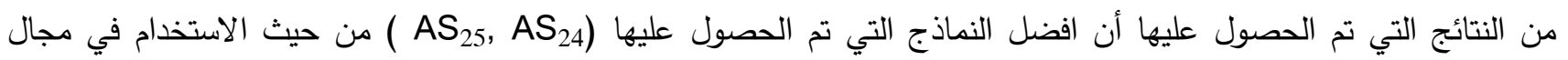
التبليط الا ان الأنموج AS يمتلك درجة ليونة تمثل الحد الادنى من القيمة المطلوبة ولذلك تم اختيار الأنموذج عن 24 كأفضل - أنموذج ولإثبات دور عملية الأكسدة الهوائية في تحسين الخواص الريولوجية تم اعادة التفاعل لأفضل النماذج التي تم الحصول عليها (AS 25, AS $_{19}$ ) الحصول عليها 
الجدول (6):الخواص الريولوجية لإفضل النماذج التي تم الحصول عليهاعند(150) م وزمن ساعة بوجود (0.5)\% وزناً من كلوريد الألمنيوم اللامائي بلون عملية الأكسدة الهوائية

\begin{tabular}{|c|c|c|c|c|c|c|c|c|c|c|c|}
\hline \multicolumn{2}{|c|}{ نسبة الاسفلتين\% } & \multicolumn{2}{|c|}{$\begin{array}{l}\text { دليل الاخترلق } \\
\text { (PI) }\end{array}$} & \multicolumn{2}{|c|}{ 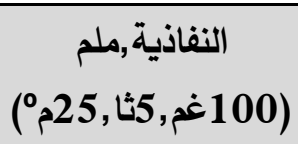 } & \multicolumn{2}{|c|}{ درجة الليونةل(مº) } & \multicolumn{2}{|c|}{$\begin{array}{c}\text { الاستطالة } \\
\text { (م)25,cm) }\end{array}$} & \multirow{2}{*}{$\begin{array}{c}\text { اللاصقبة } \\
\text { (EVA) } \\
\text { \% }\end{array}$} & \multirow[b]{2}{*}{ الأنموذج } \\
\hline المؤكسد & مؤكسد & المؤكسد & مؤكسد & المؤكسد & مؤكسد & المؤكسد & مؤكسد & المؤكسد & مؤكسد & & \\
\hline & 19.2 & & -1.413 & & 45.3 & & 50 & & $150<$ & 0 & $\mathrm{AS}_{0}$ \\
\hline 24.6 & 26.4 & -0.172 & +0.601 & 42.7 & 38.5 & 56 & 61 & $150<$ & 90 & 2 & $\mathrm{AS}_{28}$ \\
\hline 27.8 & 25.3 & -0.450 & -0.091 & 41.5 & 40.3 & 55 & 57 & $150<$ & $150<$ & 3 & $\mathrm{AS}_{29}$ \\
\hline
\end{tabular}

AS

اذ نلحظ من الجدول أعلاه ان النموذج (AS (AS)الذي تم الحصول عليه باستخدام اكسدة هوائية والمشار اليه في الجدول (4) أفضل من النموذج ( AS 28 الذي تم الحصول عليه بدون اكسدة هوائية وهذا يبين دور عملية الأكسدة الهوائية في تحسين الخواص الريولوجية للإسفلت المحور كذلك نلاحظ من الجدول أعلاه من خلال النموذج AS وكذلك النموذج 25 المشار اليه في الجدول (5) أنه بالإمكان استخدام اللاصق(EVA) المذاب في هذه النسبة وبهذا المسار في التحوير في الريولوجي باستخدام عملية أكسدة هوائية أو بدونها , مما يدل على ان عملية اختيار اللاصق كان اختيارا موفقاً وخاصة

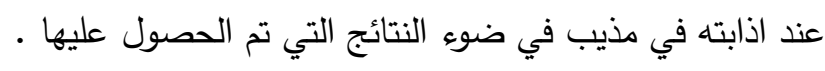
تعد عملية الأكسدة الهوائية واحدة من أهم العمليات التي تستخدم في التفاعلات العضوية على النطاق المختبري أو النطاق الصناعي

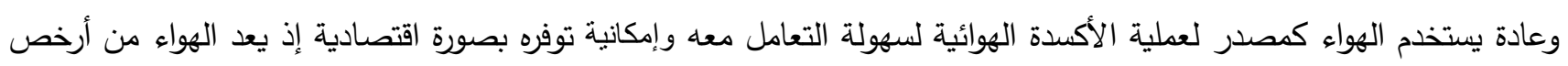
عوامل الاكسدة ، إذ إنه لايحتاج إلا إلى تكاليف قليلة تتضمن كيفية التعامل معله .

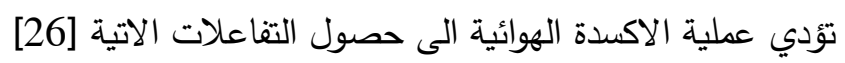

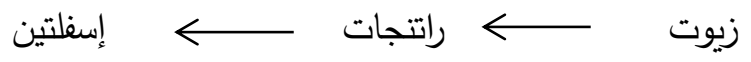

إذ يمكن القول أن الراتتجات تمثل حلقة وصل بين الإسفلتينات ذات الأوزان الجزيئية الأعلى والزيوت ذات الأوزان الجزيئية الأقل من

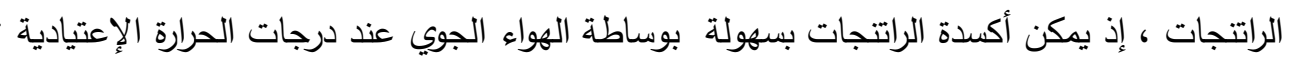
اما دليل النفاذية (PI) فيستخدم لمعرفة حساسية الإسفلت لدرجات الحرارة ,وهو عبارة عن علاقة تربط بين درجة ليونة الأنموذج الأسفلتي ودرجة نفاذيته عند (25مº) ويمكن حسابه من العلاقة الرياضية الآتية [27] $\frac{20-P I}{10+P I}=50\left[\frac{\log 800-\log p e n t}{T \cdot R \cdot B-T}\right]$

PI T:Pent

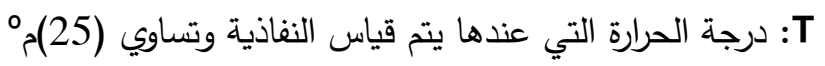

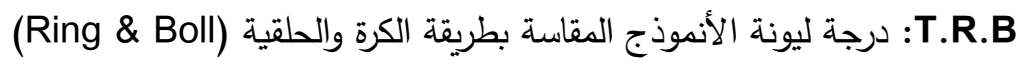


يجب ان تمتلك النماذج الاسفلتية ذات المواصفات الملائمة للاستخدام في مجال التبليط دليل اختراق ذا قيمة تقع بين (28 (2+ )

بعد ملاحظة النتائج التي تم الحصول عليها نجد أن هناك بعض النماذج المعالجة يمكن استخدامها كإسفلت تبليط بعد

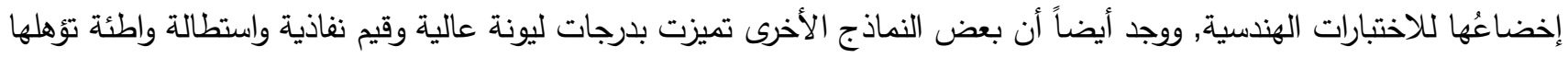

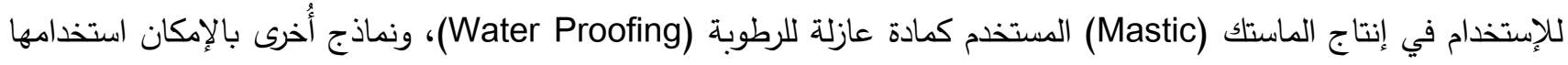
كإسفلت يستعمل في التسطيح ,كما هو موضح في الجداول من $(9,8,7)$ (7) (الجدول

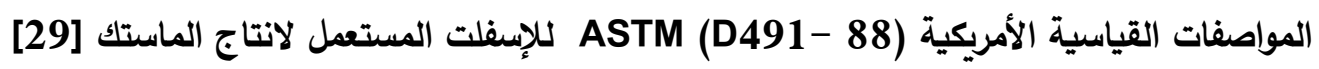

\begin{tabular}{|c|c|c|}
\hline الحد الأعلى & الحد الأدنى & القياسات الريولوجية \\
\hline 65 & 54 & درجة الليونة (مº) \\
\hline 40 & 20 & النفاذية ، ملم (100غم ، 5ثا ،25مº \\
\hline--- & 15 & الاستطالة (سم ،25م²) \\
\hline
\end{tabular}

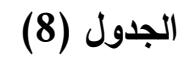

المواصفات القياسية العراقية للإسفلت المستعمل في التسطيح [30]

\begin{tabular}{|c|c|c|}
\hline الحد الأعلى & الحد الأدنى & القياسات الريولوجية \\
\hline 66 & 57 & درجة الليونة (مº) \\
\hline 40 & 18 & النفاذية ، ملم (100غم ، 5ثا ،25مº \\
\hline--- & 10 & الاستطالة (سم ،25مº) \\
\hline
\end{tabular}

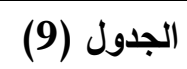

[31] (S.C.B.R) المواصفات الريولوجية للإسفلت المستعمل في التبليط حسب مواصفات هيئة الطرق والجسور العراقية

\begin{tabular}{|c|c|c|}
\hline الحد الأعلى & الحد الأدنى & القياسات الريولوجية \\
\hline 60 & 54 & درجة الليونة (مº) \\
\hline 50 & 40 & النفاذية ، ملم (100غم ، 5ثا ،25مم) \\
\hline--- & 100 & الاستطالة (سم 25ممم) \\
\hline
\end{tabular}


من أجل معرفة مدى ملاءمة النماذج الإسفلتية لأغراض التبليط ، تم إجراء فحص المارشال (التبليط بالإسفلت) لاثثين من

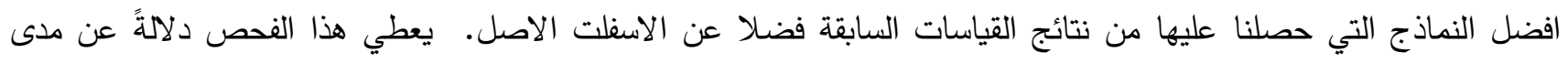

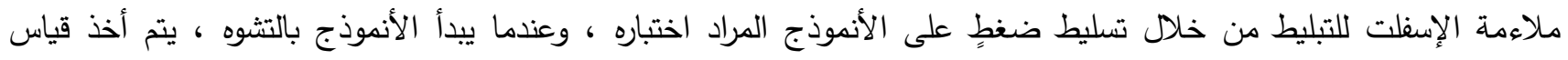
الأستقرارية (الثباتية) (Stability) والزحف (Flow) من خلال تدرجاتٍ معينة موجودةٍ في الجهاز يمكن تعريف الثباتية على أنها

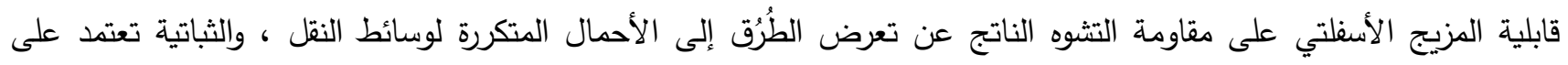

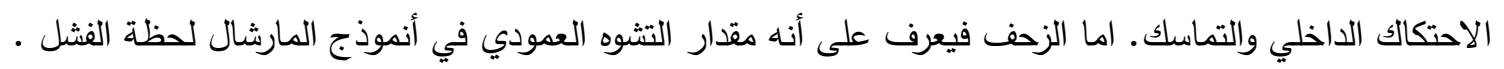

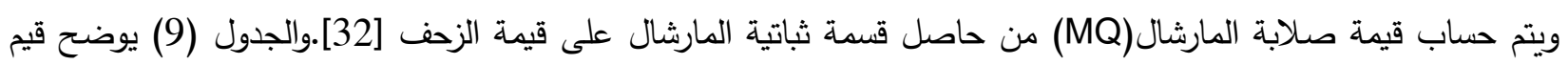

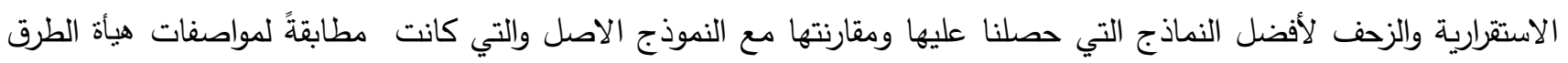
[31] (S.C.R.B) والجسور العراقية

الجدول (10) : قيم الإستقرارية والزحف للإسفلت الأصل والمحور ومواصفات هيئة الطرق والجسور (S.C.R.B)

\begin{tabular}{|c|c|c|c|c|}
\hline \multicolumn{3}{|c|}{ ألإسفلت المُحوَر } & \multirow{2}{*}{ نسبة الإسفلت المضاف إلى الركام (\%) } & \multirow{2}{*}{ 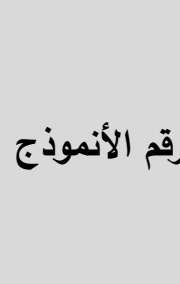 } \\
\hline MQ & $\begin{array}{l}\text { الزحف) } \\
\text { (mm) }\end{array}$ & $\begin{array}{c}\text { الإستقراريـة } \\
\text { (KN) }\end{array}$ & & \\
\hline 2.21 & 5.1 & 11.3 & \multirow{3}{*}{4.5} & $\mathrm{AS}_{0}$ \\
\hline 5.67 & 3.1 & 17.6 & & $\mathrm{AS}_{19}$ \\
\hline 6.61 & 3.4 & 22.5 & & $\mathrm{AS}_{25}$ \\
\hline $\begin{array}{c}3.5 \\
\text { الحد الأدنى }\end{array}$ & $2-4$ & $\begin{array}{c}7 \\
\text { الحد الأدنى }\end{array}$ & & AS** \\
\hline
\end{tabular}

AS** مواصفات هيئة الطرق والجسور العراقية [31] يتبين من الجدول اعلاه ان جميع النماذج المحورة كانت أفضل من الأنموذج الأصل في حال استخدامها كإسفلت تبليط , كذلك يمكن ملاحظة ان قيمة MQ للإسفلت المحور اعلى من قيمة للإسفلت الاصل وهذا يدل على ان الاسفلت المحور أكثر

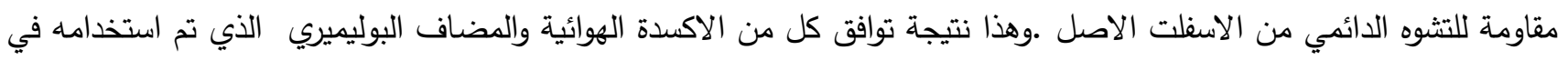
التحوير

ولأجل معرفة درجة تأثر النماذج الإسفلتية المحورة بظروف التقادم تم اجراء فحص الفرن لأغشية الأسفلت الرقيقة للأنموذج المحور ( AS 25) 
الجدول (11) : المواصفات الريولوجية للإسفلت المحور والإسفلت الأصل قبل وبعد اخضاعهما لفحص الفرن لأغثية الإسفلت (الرقيقة (TFOT)

\begin{tabular}{|c|c|c|c|c|c|}
\hline 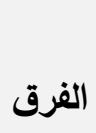 & الفحص بعد & الفحص قبل & الخواص الريولوجية & وصف الانموذج & الانموذج \\
\hline & $150<$ & $150<$ & الاستطالة سم (25م) & \multirow{5}{*}{ الأصل } & \multirow{5}{*}{$\mathrm{AS}_{0}$} \\
\hline 3 & 53 & 50 & درجة الليونة (مº) & & \\
\hline \multirow[t]{4}{*}{3.2} & 42.1 & 45.3 & النفاذية,رملم (100 غم,5ثا, 25مم) & & \\
\hline & -0.863 & -1.413 & دليل الاختراق (PI) & & \\
\hline & 0.05 & ---- & نسبة الفقدان بالوزن \% & & \\
\hline & $150<$ & $150<$ & الاستطالة (25,cmم مم) & \multirow{5}{*}{ 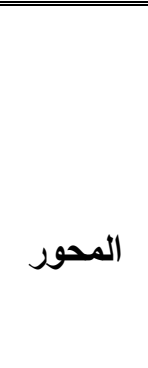 } & \multirow{5}{*}{$\mathrm{AS}_{25}$} \\
\hline 3 & 58 & 55 & درجة الليونة (مº) & & \\
\hline \multirow[t]{3}{*}{0.6} & 40.9 & 41.5 & النفاذية, ملم (100غم, 5ثا, 25م') & & \\
\hline & +0.585 & -0.450 & دليل الاختراق (PI) & & \\
\hline & 0.02 & ------- & نسبة الفقدان بالوزن \% & & \\
\hline
\end{tabular}

نلحظ من الجدول (11) ان درجة تأثر الإسفلت المحور بظروف التقادم الزمني من درجةحرارة و اوكسجين بشكل عام تكون قليلة

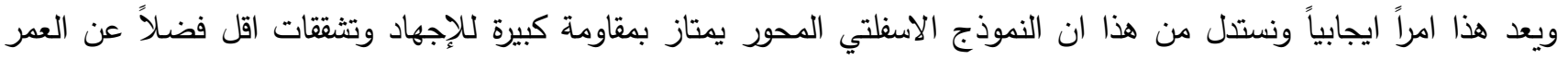

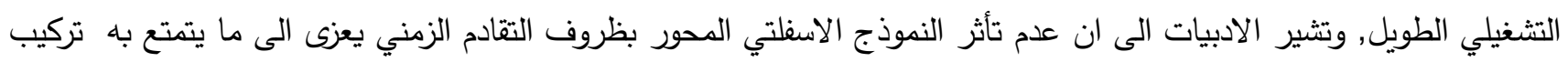

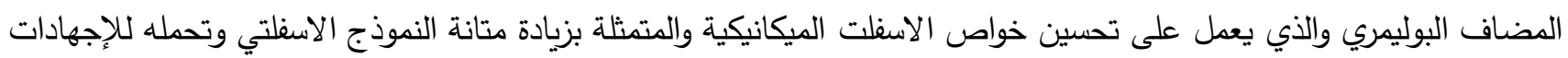

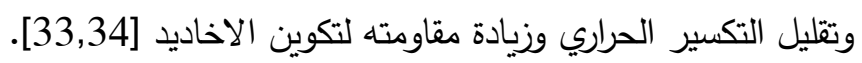
الاستنتاجات من ملاحظة النتائج التي تم الحصول عليها نستتتج : 1- إن إضافة اللاصق التجاري (EVA) إلى الإسفلت ، أثر على الخواص الريولوجية للنظام الأسفلتي بنسب متفاوتة .

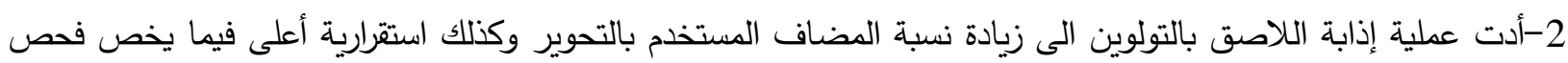
مارشال . - مار 3- أعطت هذه الدراسة قيماً لمارشال أفضل للنماذج المختارة مما هو عليه في الإسفلت الأصل وهذا يدل على إمكانية

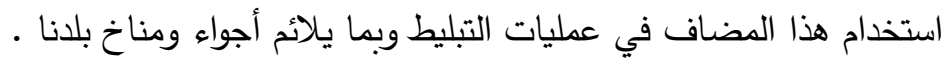
4- دلت هذه الدراسة على ان النماذج المحورة باللاصق التجاري قليلة التأثر بظروف التقادم الزمني مقارنة بالإسفلت الأصل 5 -نلحظ من الدراسة ان نسبة الإسفلتين ازدادت بصورة عامة بزيادة زمن الاكسدة وكذلك بزيادة نسبة المضاف البوليمري. 


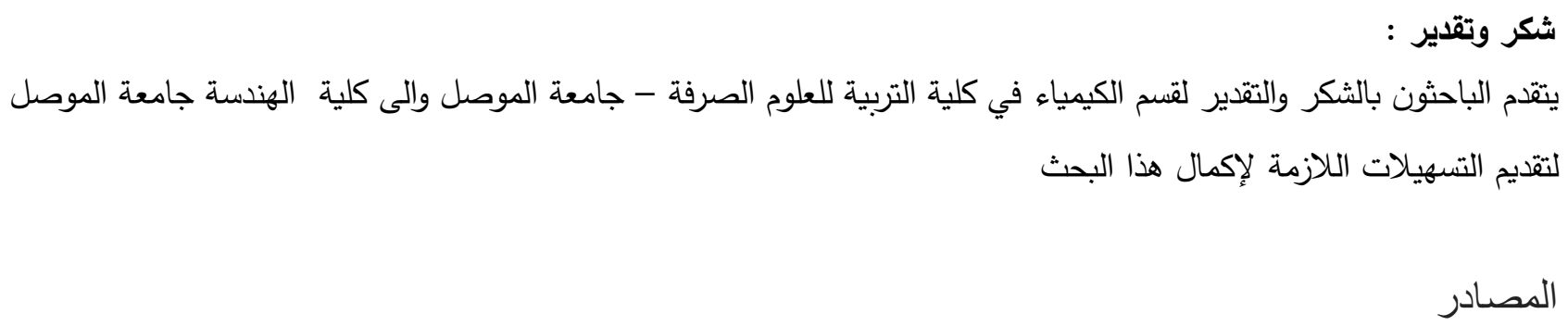

1. Al-Dabouni, A.A.; and Ali, H.," Oil of Origin, Installation and Technology. Mosul University", pp. 10-13, 63-72, 65, 69, 70-72, 166-174, 232-236, 292, 293, 294, 298, 298, 301312, 621, 622. (1986).

2. "Introduction to asphalt", Asphalt Institute Manual Series No.5(MS-5)p.2,9$11,14,61,(2001)$.

3. Parkash, S, "Petroleum Fuels Manufacturing Hand Book", McGraw-Hill Companies, Inc. 102-131., (2010).

4. Zhang, L. and Greenfield ML, Energy \& Fuels, Vol. 22, pp. 3363-3375. (2008).

5. Lesueur D., Advances in Colloid and Interface Science, pp. 145, 28-42., (2009).

6. Vargas, M. A. and Manero, O., Journal of Applied Polymer Science, Vol. 119, 2422 - 2430., (2011).

7. Hussein, A. Iqbal, H. and Al-Abdul-Wahhab, H. I., Rheol Acta, V. 45, pp. 92-104., (2005).

8. Can, J., Canadian Journal of civil Engineering, 35 (2): 148 - 157., (2008),.

9. Arslan, L., K. and Taque, ETreatment of asphalt from Heet Area Using the Residues of Basic Oils", Diala., Jour., Vol. 39., (2008).

10. Saleh, L. A., Al-Rafidain Science Journal, Volume 19, No. 2, pp. 174-190, (2008).

11. Mohammed, D. T., \& Hussein, Z. H., International Journal of Enhanced Research in S cience Technology \& Engineering, Vol. 3 Issue 9, Sept.-2014, pp: (114-121), . (2014) 
12. Gama D. A., Rosa J. M., Melo T. J. A., and Rodrigues J. K. G., Constr. Build. Mater. 106290 $-295,(2016)$.

13. Shirini, B., \& Imaninasab, R. ,Construction and Building Materials, 107, 165-171. (2016)

14. Thakre, N., Mangrulkar, D., Janbandhu, M. and Saxena, J., IOSR Journal of Mechanical and Civil Engineering Vol. 13, Issue 6 ver, PP. 120-128, (2016),

15. Hussein, A.A., Hamdoon, A.A., Journal of Education and Science, Volume (28), No. 40-49, (2019),.

16. Ali L. H., Al- Ghannam K. A. ,J. Fuel, Vol. 60, PP. 1043 - 1046., (1981),

17. ASTM, (D113 - 07), (2008).

18. STAM, , (D36-95),(2000)

19. STAM, (D5/ D5M -13), (2013).

20. ASTM, (D1559 - 2004). Standard specification, section 4, Vol., $04-03$.

21. ASTM D1754-97R "Standard test method for effect of heat and air on asphaltic materials (Thin Film Oven Test)",Section 4,Vol .04 .03,Road and paving materials-vehicle-pavement systems, New york, Ny (2002).

22. Yoder, E. J. and Witczak, M. W. "principle of pavement design", $3^{\text {rd }}$ ed., Jhon Willy and Sons., pp. 269-27, (1964),

23. Whelan, A.; Whelan, Tony. "Polymer Technology Dictionary" Springer Science \& Business Media - via Google Book ,(1994).

24. Reyes-Labarta, J.A.; Marcilla, A)., Complete Kinetic Modelling and Analysis". Industrial \& Engineering Chemistry Research. 51 (28): 9515-9530. doi:10.1021/ie3006935. (2012)

25. Flanagan, T. P., Washington, DC: U.S. Patent and Trademark Office. No. 3,448,178. (1969).

26. Al-Adam K. A, Joseph S, S, Rida M. M “Industrial Organic Chemistry”, University of Basra, pp. 94-97, (1980), 
27. Al- Frakh and Abu Shihada, A., Brit, UK Pat. App1 GB2, 028, 406, Chem. Abs. Vol. 96, $126085 \mathrm{~g}(1982)$.

28. Hobson, G. D. "Modern Petroleum Technology", $4^{\text {th }}$ ed, Ltd. Britain, PP. $804-806$, (1973),

29. ASTM D491-88, “Specification for Asphalt Mastic used in waterproofing (withdrawn 1988), withdrawn standards until (2006)", New York, N.Y.

30. Standard Specifications No. 1196 of 1988 issued by the Central Agency for Measurement and Quality Control of the bitumen used for flatness.

31. State Cooperation of Road \& Bridges (S.C.R.B). Iraqi Standard Specification, Ministry of Housing and Construction . Department of Design and Study, Section R9.), (2003),

32. Zoorob, S. E. and Suparma, L. B., Cem Cencr Compos; 22: 233-242, (2000),.

33. . Becker Y.; Menderz M.P. and Rodriguez Y. , Vision Technological, Vol. 9, No. 1,pp 39$50,(2001)$,

34. Navarro F.J., Partal P. and Martinez-Boza .F, Fuel, 2041-2049,(2004), . 